\title{
Thermomechanical and dynamic mechanical properties of bamboo/woven kenaf mat reinforced epoxy hybrid composites
}

\begin{abstract}
The dimensional stability and dynamic mechanical properties on bamboo (non woven mat)/kenaf (woven mat) hybrid composites was carried out in this study. The hybridization effect of bamboo (B) and kenaf (K) fibers at different weight ratio were studied at B:K:70:30, and $\mathrm{B}: \mathrm{K}: 30: 70$ while maintaining total fiber loading of $40 \%$ by weight. The coefficient of thermal expansion (CTE) and dynamic mechanical properties of composites were analyzed by thermomechanical anlayzer (TMA), and dynamic mechanical analyzer (DMA), respectively. Positive hybridization effects were observed on $\mathrm{B}: \mathrm{K}: 50: 50$ hybrid composite with lowest CTE and highest dynamic mechanical properties among all composites. The dimensional stability were strongly influence by the fiber orientation where all composites shows prominent expansion in the transverse fibers direction but relatively low expansion in longitudinal fibers direction. Dynamic mechanical properties in term of complex modulus $\left(E^{*}\right)$, storage modulus $\left(E^{\prime}\right)$, loss modulus $\left(E^{\prime \prime}\right)$, Tan delta and Cole-Cole plot were studied. DMA results reveal that $\mathrm{B}: \mathrm{K}: 50: 50$ hybrid composite possess the highest complex modulus due to the strong fiber/matrix interfacial bonding which supported by the coefficient of effectiveness and Cole-Cole plot. Hence, it is concluded that 50:50 weight ratio of bamboo and kenaf fibers is the optimum mixing ratio to enhance both dimensional and dynamic mechanical properties of hybrid composites, and it can be utilized for automotive or building materials applications which demand high dimensional stability and dynamic mechanical properties.
\end{abstract}

Keyword: Hybrid; Thermomechanical; Thermal analysis; Thermosetting resin 